\title{
Population Dynamics and the Epidemiological Model Proposed by Severo
}

H.M. YANG, Departamento de Matemática Aplicada, Instituto de Matemática, Estatística e Computação Científica, UNICAMP, Cx.P. 6065, 13081-970 Campinas, SP, Brazil.

\begin{abstract}
We revisit the non-bilinear incidence rate model proposed by Severo, in order to introduce a derived dynamical model taking into account the heterogeneities related to environment, immunity and genetics. The properties of that model can be better understood by the means of the population dynamics theory.
\end{abstract}

\section{Introduction}

Microparasites may be thought of as those parasites which have direct reproduction - usually at a very high rates - within the host. Hosts that recover from infection usually acquire immunity against reinfection for some time, and often for life. When the transmission stages of the parasite pass directly from one host to the next, this is called direct transmission. For such infective agents, it makes sense to divide the host population into relatively few classes (compartments) of individuals, for instance, susceptible, exposed, infectious and recovered-and-immune [1].

When a quantitative epidemiology of directly transmitted infectious diseases is considered, two fundamental mathematical concepts are evoked. The first is related to the adequate contact. It is supposed, for the group of individuals concerned, that at any given instant there is a certain chance of contact between any two individuals sufficient for the transmission of the disease if one is in infective stage and other is a susceptible. However, the possibility of an infective individual actually communicates the disease to susceptible individuals in his vicinity may depend on the virulence of the microorganisms, the extend to which they are discharged, the natural resistance of the susceptible individuals, the degree of proximity, and so on. The other concept relates to the mass action principle. The chance of a new case of infection in a very short interval of time is jointly proportional to the length of the interval and the numbers of susceptible and infectious individuals [3].

The mass action principle implies that in the community all susceptible and infectious individuals mix together homogeneously. This homogeneously mixing of individuals, at a first approximation, is most nearly realized in small household groups, but it is clearly at variance with the observed facts of social behaviour in a large town. In a homogeneous mixing assumption, all the local details - school groupings, family size, geographical location, social habits - are averaged out, and 
epidemiological and demographic processes are treated as occurring at rates that depend only on the average number or density of susceptible, infectious and immune individuals. Therefore, the difficulty of applying this approach should always be borne in mind when one is trying to deal with processes in large communities [3]. However, tests against data lead us to the conclusion that inhomogeneities associated with age-specific differences in contact rates, geographical location (cities versus village), social and cultural factors (family size, number of sexual partners) and genetic heterogeneity within the host population (leading to 'carriers' versus normal infectives) may be important in particular applications.

Anderson and May [1] assessed the effects of the foregoing aspects under a slightly modified homogeneous mixing assumption. In a different way, Severo [5] [6] introduced a great modification in the homogeneous mixing assumption. Instead of a direct product between the numbers of susceptible and infectious individuals, he considered that the probability of a new infection might be expressed as the product between the number of susceptible individuals with power $1-b$ and the number of infectious individuals with power $l$. The parameters $l$ and $b$ were called the 'infection power' and the 'safety-in-numbers power', respectively. Liu et al. [4] analyzed the stability conditions of the non-bilinear incidence rate similar to that proposed by Severo, except that the power $1-b$ was replaced by $q$.

In this paper we survey the effects of three inhomogeneities associated with the host population and the environment in the evolution of the epidemics in a community. The first is the immunological memory, which is clearly an important topic to consider when interaction occurs between host and parasite, both at the individual and at the population level. For example, a major determinant of the transmission dynamics of most microparasitic organism is the degree of specific population immunity induced by either natural infection or vaccine. This herd immunity protects directly the immune individuals from reinfection, but also confers an indirect protection to susceptible individuals. The second is the spatial non-uniformly distribution of the population [2], in such a way that the rates of transmission of an infectious disease are significantly higher in some place than in others. For instance, the environment (demographic, geographic and climatic) conditions may favor or not the spread out of the disease. Finally, the third is the genetic heterogeneity. For example, the carriers, which are 'silent' or inapparent individuals harbouring virus or bacterium and infectious individuals in the asymptomatic stage, evade the sanitary vigilance and can make contacts with other people, highly complicating both the epidemiology and the control. This non-uniformly distribution in space of carriers increases the risk of infection in the population.

This paper is divided as follows, in order to deal with the inhomogeneities. In section 2, we present a summary of the non-bilinear incidence rate modeling results (subsection 2.1), which are compared with a simple application of the population dynamics theory (subsection 2.2). Based on these results, in section 3 we develop a model considering a variable force of infection, which depends on the dynamical variables, and a brief conclusion is presented in section 4 . 


\section{The Non-Bilinear Incidence Rate Modeling}

To develop a model encompassing the herd immunity, the non-uniformly distribution in space and the genetic heterogeneity of the individuals, it is worth to understand the stochastic model proposed by Severo [5]. Since Liu et al. [4] analyzed the deterministic version of the stochastic model proposed by Severo, we summarize their results for the SEIR (Susceptible-Exposed-Infectious-Recovered) model.

\subsection{Outline}

The deterministic model proposed by Liu et al. [4], in terms of the fractions of susceptible $(s)$, exposed $(e)$, infectious $(i)$ and recovered $(r)$ individuals, is

$$
\left\{\begin{aligned}
\frac{d}{d t} s & =\mu-\beta i^{l} s-\mu s \\
\frac{d}{d t} e & =\beta i^{l} s-(\sigma+\mu) e \\
\frac{d}{d t} i & =\sigma e-(\gamma+\mu) i \\
\frac{d}{d t} r & =\gamma i-\mu r
\end{aligned}\right.
$$

where $\sigma, \gamma$ and $\mu$ are, respectively, the latent, recovery and mortality rates; $\beta$ is the contact rate, $l$ is a positive parameter and $s+e+i+r=1$, with the form $\beta i^{l} s$ being introduced to take into account the fact that the incidence rate could increase more gradually than linear in $i$ and $s$. For instance, if the number of infectives is very high, so that exposure to the disease agent is virtually certain, the incidence rate will respond slower than linearly with respect to $i$. In contrast, a rate of increase faster than linear would be observed under various conditions, for example, if multiple exposure to the disease vector were necessary before infection occurred.

For the trivial equilibrium point, given by $s^{*}=1$ and $e^{*}=i^{*}=r^{*}=0$, the stability analysis resulted in

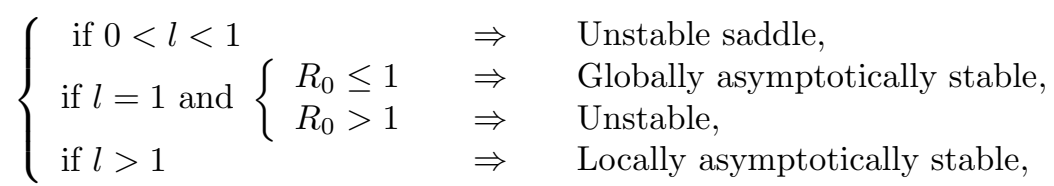

where the basic reproduction ratio $R_{0}$ is given by

$$
R_{0}=\frac{\beta \sigma}{(\sigma+\mu)(\gamma+\mu)} .
$$

The stability analysis (regarded to the trivial and non-trivial equilibrium points) remains valid for any value of the safety-in-numbers power $q$. For this reason we considered $q=1$ in the system of equations (2.1). It is important to note that the trivial equilibrium point changes its stability (from unstable to stable) with the increasing in the infection power $l$.

The non-trivial equilibrium point, designed as $s^{*}, e^{*}, i^{*}$ and $r^{*}$, can be determined. The fraction of infectious individuals $i^{*}$ is the solution of the equation

$$
\frac{R_{0}}{i^{1-l}}\left(1-\frac{i}{R}\right)=1
$$


where $R=\sigma \mu /[(\sigma+\mu)(\gamma+\mu)]$. This solution for $i^{*}$ provides the other fractions by the equations $s^{*}=R_{0}^{-1}\left(i^{*}\right)^{1-l}, e^{*}=\frac{\gamma+\mu}{\sigma} i^{*}$ and $r^{*}=\frac{\gamma}{\mu} i^{*}$.

The stability analysis for the non-trivial equilibrium point provides that

$\left\{\begin{array}{l}\text { if } 0<l<1 \\ \text { if } l=1 \text { and }\left\{\begin{array}{lll}R_{0} \leq 1 & \Rightarrow & \text { One locally asymptotically stable point, } \\ R_{0}>1 & \Rightarrow & \text { One unstable point, }\end{array}\right. \\ \text { if } l>1 \text { and }\left\{\begin{array}{lll}R_{0}<H^{-1} & \Rightarrow & \text { There is not non-trivial equilibrium point, } \\ R_{0}=H^{-1} & \Rightarrow & \text { One unstable point, } \\ R_{0}>H^{-1} & \Rightarrow & \text { Two non-trivial points, }\end{array}\right.\end{array}\right.$

where the auxiliary function $H$ is given by $H=\frac{1}{l}\left(\frac{l-1}{l} R\right)^{l-1}$. For $l>1$ and $R_{0}>H^{-1}$, the small non-trivial equilibrium $\left(i_{1}^{*}\right)$ is a unstable saddle and the large non-trivial equilibrium $\left(i_{2}^{*}>i_{1}^{*}\right)$ may be node (stable or unstable) or limit cycle (stable or unstable). We note that the results related to the homogeneously mixing assumption can be retrieved by setting $l=1$ in the system of equations (2.1). It is important to note that the large non-trivial equilibrium point changes its stability (from stable to unstable) with the increasing in the infection power $l$.

Note that a linear model is obtained if we set $l=0$ in the system of equations (2.1). In this case the trivial equilibrium point does not exist, and the unique nontrivial equilibrium point is always stable. This model does not present threshold conditions, and may be used to describe a large group of non-transmissible diseases. However, the trivial equilibrium point appears if $l$ departs from zero, whose stability goes from unstable to stable with the increasing $l$. The turning value is $l=1$, in which case we have the classical result where $R_{0}$ alone plays the role of its stability.

The unique solution $i^{*}$ of the equation $(2.3)$ decreases with increasing infection power $l$ on the interval $0 \leq l \leq 1$, which has the maximum value given by $i^{*}=\mu R_{0} /(\beta+\mu)$, when $l=0$. For $l>1$ the equation (2.3) has none, one or two solutions, according to the threshold infection power $l^{c}$, which is obtained as the maximum value of the function $f(i)=i^{l-1}(1-i / R)$. When $1<l<l^{c}$, we have two solutions $i_{1}^{*}$ and $i_{2}^{*}$, where the first increases while the second decreases monotonically with increasing $l$. The small $i_{1}^{*}$ assumes values near zero and increases abruptly to the maximum value. At $l=l^{c}, i_{1}^{*}$ and $i_{2}^{*}$ encounter and assume equal value, and above this critical value $\left(l>l^{c}\right)$ we do not have any positive solution. Specially, at $l=1$, we have the same value for the unique $i^{*}$ (for $l<1$ ) and the large $i_{2}^{*}$ (for $l>1$ ), showing a continuity between both solutions. Remember that $i_{1}^{*}$ is always unstable, while $i_{2}^{*}$ is stable for $1<l<l^{\prime}$, and unstable when $l^{\prime}<l<l^{c}$, where $l^{\prime}$ is the root of the equation $f(l)=R_{0} i_{2}^{2}-R(l-1)+1-l \gamma /(\gamma+\mu)=0$, with $i_{2}$ being a solution of the equation (2.3).

For instance, let us consider the rubella infection, which has the corresponding parameters (in years ${ }^{-1}$ ) given by $\beta=316, \sigma=52, \gamma=39$ and $\mu=0.017$. For this set of values, the threshold infection power is $l^{c}=1.199$. Numerically, the monotonically decreasing continuous function with respect to $l$, formed first by the unique solution $i^{*}$ (for $0 \leq l<1$ ) and followed by the solution $i_{2}^{*}\left(\right.$ for $1<l<l^{c}$ ), is practically unchanged until $l \sim 0.8$, and after this value the function decreases 
abruptly until $l^{c}$. Therefore, the dynamical system is strongly sensitive on the interval $0.8 \leq l \leq 1.199$, and practically insensitive on the range $0 \leq l<0.8$, which shows that the infection power acts strongly in a very narrow interval. Quite same pattern is observed for different set of values of the model's parameters.

In the next subsection we analyze the dynamics of a population stratified into two stages of life, in order to shed some more lights into the results presented by the non-bilinear incidence rate modeling proposed by Severo.

\subsection{Two Stages of Life Population Dynamics}

Let us consider a population with a life cycle divided into two classes. For instance, the mosquitoes population can be divided as winged and immature (comprising egg, larvae and pupa). The dynamics of this population can be described by

$$
\left\{\begin{array}{l}
\frac{d}{d t} A=\Phi(B) B-\left(\delta+\mu_{1}\right) A, \\
\frac{d}{d t} B=\delta A-\mu_{2} B,
\end{array}\right.
$$

where $A$ and $B$ are the number of immature and winged populations, respectively; $\mu_{1}$ and $\mu_{2}$ are, respectively, the mortality rates of immature and winged population and $\delta$ is the maturation rate. The function $\Phi(B)$ is the density dependent percapita oviposition rate. This system of equations is equivalent to the second and third equations of the system (2.1), if we consider $s=1$ (replenishing of susceptible individuals who die or acquire the infection) and write the per-capita incidence as $\beta i^{l} \equiv \varkappa(i) i$, where $\varkappa(i)=\beta i^{l-1}$ is the force of infection per infectious individuals.

Let us assume that the per-capita oviposition rate is given by $\Phi(B)=\phi B^{n-1}$, with $n \geq 0$, where $\phi$ is the oviposition rate. Then, we can calculate the equilibrium points of the system of equations (2.4), and the stability of these equilibrium points can be assessed by the eigen-values of a $2 \times 2$ Jacobian matrix, given by

$$
J=\left[\begin{array}{cc}
-\left(\delta+\mu_{1}\right) & n \phi B^{n-1} \\
\delta & -\mu_{2}
\end{array}\right],
$$

evaluated at the equilibrium point under consideration.

According to the value assigned to $n$, we have four different possibilities for the equilibrium pair $\left(A^{*}, B^{*}\right)$ :

1. $n=0$. In this case we have a linear dynamical system with only one non-trivial equilibrium point given by $\left(\frac{\phi}{\delta+\mu_{1}}, \frac{\delta \phi}{\mu_{2}\left(\delta+\mu_{1}\right)}\right)$, which is always stable.

2. $0<n<1$. We have two equilibrium points: the trivial $(0,0)$ and the non-trivial $\left(\frac{\mu_{2}}{\delta}\left[\frac{\delta \phi}{\mu_{2}\left(\delta+\mu_{1}\right)}\right]^{\frac{1}{1-n}},\left[\frac{\delta \phi}{\mu_{2}\left(\delta+\mu_{1}\right)}\right]^{\frac{1}{1-n}}\right)$. The non-trivial equilibrium point is always stable due to $\operatorname{tr}\left(J^{*}\right)=-\left(\delta+\mu_{1}+\mu_{2}\right)<0$ and $\operatorname{det}\left(J^{*}\right)=$ $(1-n) \mu_{2}\left(\delta+\mu_{1}\right)>0$, where $\operatorname{tr}$ and det are the trace and the determinant of the Jacobian matrix evaluated at the non-trivial equilibrium point. For the trivial equilibrium point, we are not able to apply this method, since the Jacobian matrix cannot be evaluated at this point. Nevertheless, for $0<n<1$ 
we have $B^{n} \gg A$ in the vicinity of $(0,0)$, and the first equation of the system (2.4) can be written as $\frac{d}{d t} A \sim \phi B^{n}$; hence this point is unstable [8].

3. $n=1$. In this case we have, again, a linear dynamical system, which has only one trivial equilibrium point given by $(0,0)$. Independently of the initial conditions, this point is stable if $\phi<\frac{\mu_{2}\left(\delta+\mu_{1}\right)}{\delta}$ and, otherwise, unstable. Note that if $\phi>\frac{\mu_{2}\left(\delta+\mu_{1}\right)}{\delta}$, then the population goes to infinity.

4. $n>1$. We have two equilibrium points: the trivial $(0,0)$ and the non-trivial $\left(\frac{\mu_{2}}{\delta}\left[\frac{\mu_{2}\left(\delta+\mu_{1}\right)}{\delta \phi}\right]^{\frac{1}{1-n}},\left[\frac{\mu_{2}\left(\delta+\mu_{1}\right)}{\delta \phi}\right]^{\frac{1}{1-n}}\right)$. The trivial equilibrium point is always stable due to $\operatorname{tr}\left(J^{*}\right)=-\left(\delta+\mu_{1}+\mu_{2}\right)<0$ and $\operatorname{det}\left(J^{*}\right)=\mu_{2}\left(\delta+\mu_{1}\right)>0$. Contrarily, the non-trivial equilibrium point is always unstable due to $\operatorname{tr}\left(J^{*}\right)=$ $-\left(\delta+\mu_{1}+\mu_{2}\right)<0$ and $\operatorname{det}\left(J^{*}\right)=-(n-1) \mu_{2}\left(\delta+\mu_{1}\right)<0$. This non-trivial equilibrium point is the so called breaking point. If the initial conditions are such that they are below the breaking point then the population goes to the extinction; otherwise, the population goes to infinity.

In summary, when $0 \leq n<1$, the oviposition rate $\Phi(B)$ maintains the size of the population always at a finite level. In another words, if the size of the population decreases, then the oviposition is greatly increased, while if the population increases so much, the oviposition is greatly decreased $\left(\Phi(B)=\frac{\phi}{B^{1-n}}\right)$. Hence, the power $n$ mimics the effects of a limiting amount of the available food. On the other hand, when $n \geq 1$, the oviposition is strongly dependent on the size of the mature individuals $\left(\Phi(B)=\phi B^{n-1}\right)$, for this reason the population either goes to extinction or increases indefinitely, depending on the initial conditions supplied to the dynamical system. In this case, the power $n$ mimics the effects of the mating function in the growth of the population, which increases with increasing number of individuals in the population. For this reason, trivial equilibrium point is always stable.

Comparing these results with those provided by the model proposed by Severo, we can observe that the per-capita force of infection $\varkappa(i)$ plays exactly the same role of the per-capita oviposition rate $\Phi(B)$, for $l<1$. Therefore, if the infection power $l$ is small, then the disease tends to be maintained at an endemic level in the community, because great numbers of infectious and immune individuals are avoided. This is true for less infective diseases, like rubella and measles. For $l \geq 1$ (high infective diseases), the difference comes out due to the first equation of the system (2.1), which constrains the population to be constant. In that epidemiological model, the stable non-trivial equilibrium point $\left(i_{2}^{*}\right)$ arises due to the role of the susceptible individuals, which appears multiplying the incidence rate in the dynamical system, which avoids in some extent (for $l \approx 1$ ) the self eradication of the disease after infecting all individuals. The low non-trivial equilibrium point $\left(i_{1}^{*}\right)$ acts as the breaking point. But, when both non-trivial equilibrium points are unstable, the dynamical system goes to the exhaustion of the susceptible individuals. For this reason first appear both breaking point and a stable equilibrium point $\left(1<l<l^{\prime}\right)$, followed by two breaking points $\left(l^{\prime}<l<l^{c}\right)$, and, then, the 
disappearing of the non-trivial equilibrium points $\left(l>l^{c}\right)$. Epidemiologically, in the first case, the endemicity is dependent on the initial conditions (e.g., introduction of a minimum quantity of infectious individuals); in the second case, the disease exhausts all susceptible individuals depending on the initial conditions; while in the last case, the disease exhausts all susceptible individuals independent of the initial conditions.

Therefore, the model considering a non-bilinear incidence rate, with the nonlinearity restricted only to the infectious individuals, is more suitable to describe an infection directly transmitted than the bilinear incidence rate modeling. In the next section we develop a model based on this dynamical behaviour, letting the infection power $l$ to vary with respect to the dynamical variables.

\section{The Variable Force of Infection Model}

From the results presented in the preceding section, we observe, always, that the locally asymptotically stable equilibrium point is the non-trivial $\left(s^{*}, e^{*}, i^{*}, r^{*}\right)$, when $0<l<1$, and the trivial equilibrium point $(1,0,0,0)$, when $l>1$. This behaviour was obtained considering non-linear per-capita force of infection $\varkappa(i)$ and constant infection power $l$. Hence, instead of setting a constant value for the infection power $l$, we let it vary according to the dynamical variables $s, i$ and $r$. We drop out the latent individuals $e$ by letting $\sigma \rightarrow \infty$, since this parameter influences weakly the behaviour of the dynamical system [7]. The assumptions that regulate the dependency of the infection power, now called $\theta$, with the dynamical variables are:

1) the spatial heterogeneity and the herd immunity contribute to drive the dynamical system to the trivial equilibrium point. We assume that the protection given by herd immunity obeys a linear relation with respect to $r$, while the constant term represents the natural barrier, so $\theta_{p}(r)=p_{0}^{\prime}+p^{\prime} r$, and

2) the risk represented by the available susceptible individuals and the carriers (due to the genetic heterogeneity) contribute to drive the dynamical system to the non-trivial equilibrium point. We also assume a linear relation for this form of risk with respect to $s$ and $i$, setting $\theta_{h}(s, i)=a s+h^{\prime} i$.

Therefore, taking into account the above two assumptions, that is, the immune individuals plus the environment (healthy or polluted) can be thought of as barriers against the dissemination of the infection, while the susceptible and infectious individuals can be considered to facilitate the transmission of the virus, the infection power $\theta$ can be described by

$$
\theta(s, i)=\frac{p_{0}+p(1-s-i)}{s+h i},
$$

where we have $p_{0}=p_{0}^{\prime} / a, p=p^{\prime} / a, h=h^{\prime} / a$, and $r=1-s-i$. The parameters $p$ and $p_{0}$ are protection degrees conferred by the herd immunity and the environment, respectively, and $h$ is the hazard degree due to the carriers distributed inhomogeneously in the community. The protection degrees measure how the immune 
individuals and the environment are opposing to an adequate contact, whereas the hazard degree is a measure of the ability of the infection being transmitted whenever an adequate contact is made among susceptible and infectious individuals.

The generalized non-bilinear incidence SIR modeling can be written as

$$
\left\{\begin{aligned}
\frac{d}{d t} s & =\mu-[\lambda(s, i)+\mu] s, \\
\frac{d}{d t} i & =\lambda(s, i) s-(\gamma+\mu) i,
\end{aligned}\right.
$$

where the decoupled fraction of recovered individuals is obtained from $r=1-s-i$, and $\lambda(s, i)=\beta i^{\theta(s, i)}$ is the variable non-linear force of infection.

The system of equations (3.2), with the non-linear force of infection given by $\lambda(s, i)=\beta i^{\theta(s, i)}$, presents two asymptotic situations. The first is the disease free population, given by the trivial equilibrium point, and the other, the disease at an endemic level in a population, given by the non-trivial equilibrium point(s). In this paper we restrict to the analysis of the trivial equilibrium point.

By inspecting the system of equations (3.2), we observe that the trivial equilibrium point $(1,0)$ is always possible. The stability of this trivial solution is provided by analyzing the system of infection (3.2) in the vicinity of the equilibrium point $(1,0)$. Rewriting the fraction of infectious individuals given by the equation (3.2) as

$$
\frac{d}{d t} i=\left[\beta s i^{\frac{p_{0}-1+(p+1) r-(h-1) i}{1-r+(h-1) i}}-(\gamma+\mu)\right] i \leq \beta\left[i^{\frac{p_{0}-1+(p+1) r-(h-1) i}{1-r+(h-1) i}}-R_{0}^{-1}\right] i,
$$

because we have $1 \geq s(=1-i-r)$; and $R_{0}=\beta /(\gamma+\mu)$, by letting $\sigma \rightarrow \infty$ in the equation (2.2). We observe that the stability depends on the sign of the exponent. In other words, since $1-r+(h-1) i>0$, if

$$
p_{0}-1+(p+1) r-(h-1) i \geq 0,
$$

then the trivial equilibrium point can be stable and, otherwise, is always unstable.

First, let us understand the role of the environment related parameter $p_{0}$. Depending on the value assumed by $p_{0}$, the upper semi-plane (for simplicity, restricted to $h>1$ ), that obeys the inequality (3.4), contains or not the origin (which is the trivial equilibrium point). Hence, the inequality (3.4) is a necessary condition to have the stability of the trivial equilibrium point. To be more clear, in the $i \times r$ plane, the semi-region positively defined contains the trivial equilibrium if $p_{0} \geq 1$. Hence, if $p_{0} \geq 1$, then $(1,0)$ can be stable. Otherwise, the semi-region positively defined does not contain the trivial equilibrium $(1,0)$. Hence, if $0<p_{0}<1$, then $(1,0)$ is always unstable. Observe that on the line equation $p_{0}-1+(p+1) r-(h-1) i=0$ the classical result is retrieved, that is, if $R_{0} \leq 1$, then $(1,0)$ is stable, only when $p_{0} \geq 1$. Therefore, the disease cannot be eradicated in a polluted environment (given by $0<p_{0}<1$, which means individuals living in a community with very bad socio-economic and sanitation conditions).

Second, let us survey the sufficient condition under which the disease can be established in a community when one infection is introduced in the community. For this purpose, we consider the equation (3.3) in the situation $r=0$, that is,

$$
\frac{d}{d t} i \leq \beta\left[\Omega(0)-R_{0}^{-1}\right] i,
$$


where $\Omega(0)$ (from the equation (3.3) with $r=0$, corresponding to the time of the introduction of an infection in the community) is the supremum in $i$, given by

$$
\Omega(0)=\sup _{0<i<i_{m}}\left\{i^{\frac{\left(p_{0}-1\right)-(h-1) i}{1+(h-1) i}}\right\}
$$

with $i_{m}=R$, according to the equation (2.3). Therefore, the trivial equilibrium point is globally asymptotically stable whenever we have $\Omega(0) \leq R_{0}^{-1}$, otherwise, we cannot conclude anything. Observe that the exponent is always negative if $0<$ $p_{0}<1$ and if $p_{0}>1$ with $i>i_{c}$ (of course, $\left.i_{c} \leq i_{m}\right)$, where $i_{c}=\left(p_{0}-1\right) /(h-1)$. In both cases the trivial equilibrium point is always unstable and the disease can be established in the community. The exponent, on the other hand, is positive if $p_{0}>1$ and $i \leq i_{c}$, in which case the disease may not be established in the community.

In the case $p_{0}>1$ and $i \leq i_{c}$, we must take into account the value of $\Omega(0)$ to assess the stability of the trivial equilibrium point, because the exponent is always positive and the function $(i)^{\frac{\left(p_{0}-1\right)-(h-1) i}{1+(h-1) i}}$ is monotonically increasing. Therefore, if $\Omega(0) \leq R_{0}^{-1}$, then the disease cannot be established. However, if $\Omega(0)>R_{0}^{-1}$, then we have a threshold fraction of infectious individuals $i^{\text {th }}$ obtained as a solution of

$$
\left(i^{t h}\right)^{\frac{\left(p_{0}-1\right)-(h-1) i t h}{1+(h-1) i^{t h}}}=R_{0}^{-1}=\Omega^{*},
$$

such that for $i>i^{t h}$ the disease can be established. In another words, the disease can be established if the initial fraction of infectious individuals is higher than its threshold value. Observe that $i^{t h}$ is diminished if $R_{0}$ is increased.

To illustrate the above findings, let us consider again the rubella infection. The set of previously given values resulted in $R_{0}=8.1$ and $R_{0}^{-1}=0.123472$. Numerically, if we consider $p_{0}=2, p=0.01$ and $h=50,000$ for the parameters related to the infection power, then the disease fades out if $i_{0}=1.37043 \times 10^{-5}$ $\left(\Omega^{*}=0.123469\right)$ and it is established in the community if $i_{0}=1.37047 \times 10^{-5}$ $\left(\Omega^{*}=0.123491\right)$. From the sufficient condition, that is, using the equation (3.7), we obtain $i^{\text {th }} \simeq 1.37045 \times 10^{-5}$, which situates between those two values. On the other hand, for lower value of $h$, for instance $h=10,000$, the disease fades out if $i_{0}=6.43814 \times 10^{-5}\left(\Omega^{*}=0.123490\right)$ and it is established in the community if $i_{0}=6.43819 \times 10^{-5}\left(\Omega^{*}=0.123494\right)$. The equilibrium values corresponding to the first case is $s^{*}=0.0001$ and $i^{*}=0.0004$; while for the second case we have $s^{*}=0.03$ and $i^{*}=0.0000001$. Dynamically, in both cases the disease practically exhausts susceptible individuals in the first few moments; but in the second case (due to the lower value for $h$ ) there is the resurgence of the susceptible individuals due to the very low fraction of infectious individuals.

\section{Conclusion}

We applied the population dynamics theory to understand the non-homogeneous epidemiological model proposed by Severo. Due to the non-linearity, this model is not well understood in its dynamics and in the stability analysis of the equilibrium 
points. From the results obtained using a very simple population dynamics model, we clarified some aspects of the dynamics and the stability of the equilibrium points of the epidemiological model proposed by Severo.

Based on this understanding, we proposed a variable non-linear force of infection to analyze the onset of the epidemics diseases in the community taking into account heterogeneities like environment, immunity and genetics. Even that we restricted to the analysis of the trivial equilibrium point, the dynamics of the infections described by the system of equations (3.2) provided us with some interesting epidemiological findings. For instance, very infective diseases, like common cold and influenza, cannot be maintained in the community due to the exhaustion of the susceptible individuals.

Resumo. Considera-se o modelo de incidência não-bilinear proposto por Severo com a finalidade de entender a sua dinâmica e estudar os efeitos de heterogeneidades tais como meio-ambiente, imunidade e fatores genéticos. O primeiro objetivo é alcançado por meio da teoria de dinâmica populacional, enquanto o segundo é obtido utilizando-se uma força de infecção não-linear dependente das variáveis dinâmicas.

\section{References}

[1] R.M. Anderson and R.M. May, "Infectious Diseases of Humans - Dynamics and Control", Oxford University Press, Oxford, New York, 1992.

[2] N.T.J. Bailey, Stochastic birth, death and migration processes for spatially distributed population, Biometrika, 55 (1968), 189-198.

[3] N.T.J. Bailey, "The Mathematical Theory of Infectious Diseases and Its Applications", Charles Griffin and Company Ltd., $2^{\text {nd }}$ Ed., London and High Wycombe, 1975.

[4] W.M. Liu, H.W. Hethcote and S.A. Levin, Dynamics behavior of epidemiological models with nonlinear incidence rates, J. Math. Biol., 25 (1987), 359-380.

[5] N.C. Severo, Generalizations of some stochastic epidemic models, Math. Biosc., 4 (1969), 395-402.

[6] N.C. Severo, The probabilities of some stochastic epidemic models, Biometrika, 56 (1969), 197-201.

[7] H.M. Yang, Modelling vaccination strategy against directly transmitted diseases using a series of pulses, J. Biol. Systems, 6, No. 2 (1998), 187-212.

[8] H.M. Yang and W.C. Ferreira Jr., A population model applied to HIV transmission considering protection and treatment, IMA J. Math. Appl. Med. Biol., 16, No. 3 (1999), 237-259. 